\title{
Generation Youtube: Die Zerstörung der Politik oder die Fortsetzung von Politik mit anderen Mitteln ${ }^{1}$
}

\author{
Valentin Dander
}

\section{Zusammenfassung}

Im Beitrag wird der Zusammenhang von YouTube-Nutzung Jugendlicher und gegenwärtiger politischer Kultur analysiert. Ausgehend von Rezos YouTube-Video „Die Zerstörung der CDU“ geht der Text der Frage nach, wie Jugendliche das Internet und insbesondere Videoplattformen für ihre Informationsbeschaffung und Meinungsbildung nutzen. YouTube wird demnach als ein Ort politischer Information und Bildung begriffen, der insbesondere das Visuelle bedient. In Diskussionen über das Rezo-Video wurde verstärkt die Verschiedenheit digital-politischen Handelns zwischen der jungen und der älteren Generation als Differenzlinie eingezogen. Im Text wird hingegen dargelegt, dass und inwiefern politische Medienkulturen in und jenseits von Generationenverhältnissen zu denken sind.

\section{Abstract}

The article analyses the connection between the use of YouTube by young people and current political culture. Based on Rezo's YouTube video "The Destruction of the CDU", the text explores the question of how young people use the Internet and in particular video platforms to obtain information and form opinions. YouTube is thus conceived as a place of political information and education that serves the visual in particular. In discussions about the Rezo video, the difference in digital-political action between

1 Dieser Text wurde im Heft 42 von ZWISCHENTÖNE - Das Generationen-Magazin an der Hochschule Niederrhein unter einer CC BY 3.0 DE Lizenz erstveröffentlicht (S. 4-11). Im Text wurden geringfügige Korrekturen vorgenommen. Ton und Form sind an eine breitere Öffentlichkeit gerichtet und wurden beibehalten. 
the younger and older generation was increasingly drawn as a line of distinction. In contrast, the text explains that and to what extent political media cultures are to be thought in and beyond generational relations.

\section{Einleitung}

Kurz vor der Europawahl 2019 wurden in der Bundesrepublik Deutschland heftige Diskussionen über die Rolle der Online-Videoplattform YouTube für das politische System geführt. Was war geschehen? Am 18. Mai 2019 veröffentlichte der 26-jährige Youtuber Rezo (2019a) auf seinem Kanal Rezo ja lol ey ein Video mit dem Titel „Die Zerstörung der CDU“. Zweieinhalb Wochen nach Veröffentlichung haben fast 15 Mio. Menschen dieses Video angeklickt. Seit 2015 produziert Rezo in erster Linie Spaß- und Musikvideos. Seine beiden Kanäle weisen in Summe mehr als 2,5 Mio. Abonnements auf (vgl. rezo o.J.). Das bedeutet, dass die Abonnent*innen informiert werden, wenn ein neues Video online gestellt wird. Bereits Ende November 2018 meldete er sich mit einem Erklärvideo zur aktuellen Urheberrechtsreform der Europäischen Union zu Wort. In dem neueren Video jedoch bezieht er sehr explizit politisch Stellung, indem er selbst dabei zu sehen ist, wie er verschiedene politische Positionen und Entscheidungen der CDU/CSU, aber auch der SPD und anderer Parteien, kommentiert. Das Video dauert fast eine ganze Stunde und ist durchsetzt von Grafiken, Videos und Referenzen, die in einem OnlineDokument nachgelesen werden können. Die Quellen, auf die sich Rezo hierbei bezieht, sind teils wissenschaftliche Studien, teils journalistische Texte, teils Originalmaterial der Parteien und anderer thematisierter Organisationen.

Im Kern besteht der Inhalt des Videos darin, Aussagen und Entscheidungen der Regierungsparteien zu Themen wie Armut, Klimawandel, Urheberrecht und Kriegsbeteiligung mit wissenschaftlichen Argumenten abzugleichen. In den meisten Fällen - insbesondere in Sachen Umwelt- und Klimapolitik - kommt er zum Schluss, dass hier eine große Diskrepanz besteht. So schließt er denn das Statement mit einer Wahlempfehlung gegen CDU, CSU, SPD und AFD und für die Ausübung des Wahlrechts bei der Wahl zum Europaparlament - insbesondere durch sein größtes Publikum: junge Menschen. „Denn die größte Wahlmacht,“ so sagt er (53:25), „haben die Alten, nicht wir. Zum Vergleich: Allein die über 70-Jährigen, also ein bestimmter Teil der Rentner, haben anderthalb Mal so viele Stimmen wie alle unter 30-Jährigen. Die Rentner entscheiden also mehr über unsere Zukunft, obwohl sie diese Zukunft gar nicht mehr miterleben werden." Er ruft nun sein Publikum dazu auf, mit ihren Eltern und Großeltern zu sprechen und sie zu bitten, ihre Stimme nicht „einer solchen lebenszerstörerischen Partei“ zu geben (54:08). Knapp eine Woche nach seinem Video folgte ein von ihm initiiertes 
Statement von mehr als 90 Youtuber*innen, das in einem knapp 3-minütigen Appell bzw. Offenen Brief den Nicht-Wahlaufruf für CDU, CSU, SPD und AFD wiederholt und v.a. mit ihrer Klimapolitik begründet (Rezo ja lol ey 2019b).

Nun stellt sich die Frage, warum diese Geschichte hier erzählt werden sollte. Auf diese Frage lässt sich auf verschiedenen Ebenen antworten - und zwar mit weiteren, daran anschließenden Fragen, die im Folgenden in Grundzügen beantwortet werden:

(1) Welche Rolle kommt solchen Videos für junge Menschen für Information und politische Meinungsbildung zu?

(2) (Inwiefern) Trifft die Aussage zu, dass wir es hier vor allem mit einer Differenz zwischen Generationen zu tun haben?

(3) In welchem Verhältnis steht diese Form der politischen Digitalkultur zum etablierten politischen Feld im Allgemeinen und welche Konsequenzen folgen daraus?

\section{YouTube als Ort politischer Information und Bildung Jugendlicher}

Im Nachgang zur Veröffentlichung und der öffentlichen Diskussion des Rezo-Videos wurde die CDU in der Öffentlichkeit unter Zugzwang gesehen. Sie müsse auf die Vorwürfe antworten. Philipp Amthor, MdB der CDU und um wenige Monate jünger als Rezo, hatte zunächst ein Antwort-Video erstellt, das allerdings nicht veröffentlicht wurde. In einem Statement gegenüber der Tagesschau begründete er diese Entscheidung der Parteispitze wie folgt: „Wir wollen keinen ewigen Austausch jetzt über Videos, sondern lieber einen Austausch auf einer Argumenten- und Sachebene." (tagesschau 2019: 01:06)

Diese Gegenüberstellung von (Web-)Videos und einer rationalen Debatte legt nahe, dass sachliche Argumente in solchen Videos nicht zu finden seien. Diese Ansicht lohnt es zu überprüfen. Zunächst kann am konkreten Beispiel des Rezo-Videos gefragt werden, wie informativ darin gearbeitet wird - gerade auch im Umgang mit den Quellen. An verschiedenen Stellen wurden ,Faktenchecks' durchgeführt, die zwar einige Verkürzungen, Zuspitzungen und auch manche unzutreffenden Aussagen identifizieren. Über weite Strecken sei Rezos Argumentation aber tragfähig (vgl. Bernau et al. 2019; Bojanowski et al. 2019; maiLab 2019). Zugleich sollte aber die Unterscheidung zwischen informierenden und kommentierenden bzw. meinungsorientierten (hier quasi-journalistischen) Beiträgen berücksichtigt werden, so schwierig diese Trennlinie auch zu ziehen ist (vgl. Rakebrand/Jünger 2013, S. 31). Rezos Video, das nicht im engeren Sinne ein journalistisches Medienprodukt ist, entspricht eher einem meinungsbasierten Beitrag. Genau diese Ausrichtung bedient die Interessen jugendlicher YouTube-Nutzer*innen, wie eine 
qualitative Studie zum Thema ausweist (vgl. Hugger et al. 2018, 2019). So beschreiben die interviewten Jugendlichen die Aspekte Meinungsorientierung und Authentizität auf YouTube als bedeutsam. Über diesen Weg kann Youtuber*innen auch eine gewisse Verantwortung zugeschrieben werden: „Das Forschungsprojekt zeigte, dass informationsorientierte YouTuber(inne)n [sic!] eine Wirkung auf politische Meinungsbildung von Jugendlichen haben“" (Gräßer et al. 2019). ${ }^{2}$

Dass die Jugendlichen politische Informationen (und Meinungen) online gezielt suchen, zeigt auch die JIM-Studie, eine jährliche, für Deutschland repräsentative Untersuchung der Mediennutzung 12-19-Jähriger. Ihr zu Folge nutzen diese Jugendlichen YouTube nicht nur im steigenden Maße (2018: 60 \% / 2016: 42 \%) mindestens mehrmals pro Woche, sondern $16 \%$ der Mädchen und $29 \%$ der Jungen der YouTube-Nutzer*innen nutzen die Plattform, um sich „Videos von Youtubern über aktuelle Nachrichten“ anzusehen (Feierabend u. a. 2018, S. 47, 50). Eine weitere Studie der bitkom deutet auf Altersunterschiede in kindlicher und jugendlicher Internet-Nutzung hin (Berg 2017, S. 7): $69 \%$ der 6-7-Jährigen schauen im Internet zumindest ab und zu Videos/Filme/ Serien, nur 14 \% suchen Informationen zu persönlichen Interessen. Bei den 16-18-Jährigen werden zwar ebenfalls von vielen Videos etc. angesehen (84 \%), der Anteil jener, die sich informieren, liegt dabei mit $64 \%$ jedoch wesentlich höher als bei der jüngeren Altersgruppe. ${ }^{3}$ Allerdings finden wir mit dieser Differenzierung - Videos schauen oder Information suchen - erneut die These von CDU bzw. Philipp Amthor, dass die beiden Tätigkeiten nicht miteinander zu verbinden seien.

Dass dem nicht so ist, das belegte bereits vor einigen Jahren eine Bremer Studie zum Lernen mit Onlinevideos. Diese seien ,alltäglich und breitgefächert genutzte Lernobjekte geworden, die Jugendliche im Internet suchen und für ihr eigenes alltägliches und schulisches Lernen verwenden." (Rummler/Wolf 2012, S. 265) In der jüngsten Studie zur YouTube-Nutzung gibt ein Viertel der 12- bis 19-Jährigen an, dass ihnen an der Plattform wichtig ist, Inhalte seien gut erklärt und erweitern ihr Wissen (vgl. Rat für

2 Dass YouTube noch vor weniger als zehn Jahren diesbezüglich kaum eine Rolle gespielt hat, zeigt eine Studie, die 2011 mit 12- bis 20-Jährigen durchgeführt wurde. In der Darstellung wird YouTube kein einziges Mal genannt. „Seiten für Videos/ Hörbeiträge“ landen mit 24,5 \% täglicher Nutzung knapp hinter „Informations- und Nachrichtenportale[n]“ (29,2\%); beide deutlich abgeschlagen hinter „Suchmaschinen“ (46,7 \%) und „Netzwerke/ Communities“ (38,4 \%). (vgl. Gebel/Jünger/ Wagner 2013: 36)

3 Die Shell-Jugendstudie von 2015 verzeichnet für Internetnutzung allgemein bei den 22- bis 25-Jährigen fast $50 \%$, die nach „Informationen suchen, die ich gerade brauche“ (Leven/ Schneekloth 2015: 143). Mehr als drei Viertel dieser Gruppe geht dieser Tätigkeit mindestens einmal pro Woche nach (vgl. ebd.). 
Kulturelle Bildung 2019, S. 19). Zudem werden Videos kontextabhängig nicht nur rezipiert, sondern in vielen Fällen auch selbst produziert (vgl. Wolf/Kratzer 2015). Wir können also festhalten, dass auch junge Menschen Videos im Internet zur Information und Meinungsbildung nutzen - und dort entsprechende Inhalte wie eben das Rezo-Video vorfinden, die dieses Bedürfnis stillen. Dabei kommt besonders kommentierenden, meinungsstarken Formaten große Bedeutung zu.

\section{Politik und Medienkultur in und jenseits von Generationenverhältnissen denken}

Die ARD/ZDF-Onlinestudie liefert repräsentative Daten für die Mediennutzung der deutschen Bevölkerung ab 14 Jahren und verzeichnet 2018 insgesamt 64 \%, die zumindest selten „Videoportale wie YouTube“ nutzen (Kupferschmitt 2018, S. 429). Hierbei zeichnet sich aber „beim Konsum von Onlinevideos ein ausgeprägtes Altersgefälle“ ab (ebd., S. 428). 98 \% der 14-29-Jährigen und $85 \%$ der 30-49-Jährigen stehen $45 \%$ bei 50-69-Jährigen und $18 \%$ bei den über 69-Jährigen gegenüber. Eine weitere Differenzierung wäre hierbei in Sachen Nutzungsfrequenz einzuziehen. In der ältesten Gruppe fällt der Zuwachs gegenüber der letztjährigen Erhebung übrigens mit fünf Prozentpunkten am höchsten aus. Wird die Frage auf Onlinevideos insgesamt ausgedehnt, geben $50 \%$ der über 55-Jährigen 2018 an, solche zu nutzen (vgl. Statista 2018). Um diese Zahlen ins Verhältnis zur Gesamtbevölkerung zu setzen: Für 2017 verzeichnet das statistische Bundesamt 21,5 \% der Deutschen mit 65 Jahren oder älter (vgl. Statistisches Bundesamt 2018).

In Bezug auf das Wahlverhalten am Beispiel der EU-Wahl 2019 können gleichfalls große Unterschiede zwischen Altersgruppen festgestellt werden, wie tagesschau.de (o.J.) durch einen Vergleich des in einer Infratest dimap-Umfrage erhobenen Wahlverhaltens von unter-25-Jährigen und der Gruppe 60 Jahre und älter zeigt. Die größten Differenzen zeigen sich an den Ergebnissen von CDU/CSU und den Grünen. Hier stehen $13 \%$ (Grüne) und $41 \%$ (Union) in der höheren Altersgruppe $34 \%$ (Grüne) und $12 \%$ (Union) bei den Jüngeren gegenüber. Die Differenz bei SPD-Wähler*innen fällt etwas geringer aus $(8 \% / 22 \%)$.

Vor diesem Hintergrund könnte vermutet werden, dass das Alter die entscheidende Variable sei, um Mediennutzungs- wie Wahlverhalten zu erklären. Weiter könnte , gedeutelt ${ }^{`}$ werden, dass hier sogar ein Zusammenhang zwischen beiden Verhaltensweisen im Internet und in der Politik bestehe. Ganz so einfach ist die Sache natürlich nicht. Die Unterschiede bezüglich des Wahlverhaltens wurden zwar von einigen Kommentator*innen 
herangezogen, um einen regelrechten Kampf der Generationen um politische Hegemonie herbeizureden. Obwohl das Alter ein Faktor bei Wahlentscheidungen ist, der einen Unterschied machen kann, gibt es weitere bestimmende Parameter, wie beispielsweise den Bildungsgrad. Laut Zahlen von merkur.de wurden die Grünen von $31 \%$ der Hochschulabsolvent*innen gewählt, die Unionsparteien hingegen nur von $22 \%$. Umgekehrt wählten $9 \%$ der Hauptschüler*innen bzw. Wähler*innen mit max. Hauptschulabschluss die Grünen und 39 \% die CDU/CSU (vgl. Strobl 2019). Die Ergebnisse einer Studie über die Teilnehmer*innen an der ökologischen Protestbewegung Fridays for Future, die v.a. von Schüler*innen getragen wird, weisen eine ähnliche Tendenz auf: hohe Bildungsabschlüsse und akademische Haushalte (vgl. Institut für Protestund Bewegungsforschung (ipb) 2019). Auch das Geschlecht, Berufsfelder etc. können herangezogen werden und jeweils würden sich deutliche Unterschiede zwischen verschiedenen Teilgruppen feststellen lassen. All das bedeutet zwar nicht, dass das Alter eine unbedeutende Kategorie sei, macht aber deutlich, dass sie eben eine von zahlreichen Kategorien ist, die lediglich in ihrem wechselseitigen Zusammenspiel zu interpretieren sind.

Was nun die medialen Formate der politischen Information und Meinungsbildung betrifft, so schienen diese auf den ersten Blick sehr verschieden zu sein. Wie der Medienwissenschaftler Christoph Engemann aber argumentiert, sind die Formate in mancher Hinsicht gar nicht so neu wie sie scheinen. Er beschreibt in einem Thread im Microblogging-Dienst Twitter das Rezo-Video im Vergleich mit klassischen Hochschulvorlesungen und stellt verblüffende Parallelen fest - gerade mit Blick auf Format, Inhalt und Länge. Zugleich bestehen natürlich Unterschiede, etwa in der Sprache (Umgangs- und Jugendsprache), der institutionellen Rahmung, des Publikums und eben auch in medialen und medienkulturellen Formen: „(12) Die neue Vorlesung erwartet Menschen, die u.a. am Lesen gebildete, komplexe Diskurse verfolgen wollen \& zugleich in der Masse und Verfügbarkeit von Texten Orientierung erwarten." (Engemann 2019) Algorithmische Selektion ist einer der Faktoren, der auf Online-Plattformen wie YouTube zur Orientierung beitrage - wenn auch nicht immer von allen Jugendlichen entsprechend reflektiert und kritisch betrachtet (vgl. Hugger et al. 2019). ${ }^{4}$

Scrollt man unter dem Rezo-Video in der Kommentarspalte nach unten, wird deutlich, dass es sich hier nicht nur um den Austausch einer homogenen Altersgruppe handelt. Ohne besonders lange suchen zu müssen, tauchen Statements von Usern auf (die ge-

4 Ein Beispiel für problematische Aspekte: Der YouTube-Algorithmus ordnete private Videos, die bspw. Kinder oder Jugendliche auf nicht-sexualisierte Weise beim Baden zeigen, gemeinsam mit explizit sexualisierten Inhalten ein (vgl. Fisher/Taub 2019). 
wählten Namen lassen überwiegend auf Männer schließen), denen es offenbar wichtig ist, ihr Alter jenseits der 60 Jahre angeben. Die unten angeführten Beispiele verdeutlichen, dass YouTube als Plattform zumindest in Teilen eine mediale und medienkulturelle Infrastruktur bereitstellt, die die Kommunikation zwischen den Generationen ermöglicht - bei aller berechtigten Kritik an intransparenten Algorithmen und den verschiedenen Monetarisierungsstrategien der Plattform und der Beteiligten.

„Dieses Video ist ein ganz großes Werk: Die perfekte Kombination von Herz und scharfem Verstand, Emotion, Courage und Fleiß. Ich bin tief beeindruckt (und 63 Jahre alt).“

„Nach meinen 66 Lebensjahren auf dieser Erde, freue ich mich, endlich mal wieder auf jemanden zu treffen, der sich traut in einem Massenmedium den Mund aufzumachen.“

„Ich bin 67 und finde es einfach genial wie du es erklärst tech ech super $\hat{\theta}$ mein Sohn hat mir es gezeigt und ich finde es einfach perfekt wie du dich dafür einsetzt $\because$ :“

(vgl. die Kommentarspalte von Rezo ja lol ey 2019a)

\section{Digitalisierung politischer Kultur?}

Die Art des Diskurses auf YouTube unterscheidet sich aber doch sehr von jenem der etablierten politischen Kommunikation, so hält Engemann in einem Interview mit dem Deutschlandfunk fest, dass der Austausch zwischen diesen Formen zu Schwierigkeiten führe (Anwar 2019): „Zwei Formen der Diskursivität versuchen miteinander ins Verstehen zu kommen." Dass diese Verständigung mitunter ,episch scheitert", ist an drei Beispielen abzulesen, die jeweils in Reaktionen von Politiker*innen auf zivilgesellschaftliches Engagement mehrheitlich Jugendlicher oder junger Erwachsener bestehen:

1. Nachdem gegen die damals noch geplante Urheberrechtsnovelle der Europäischen Union (vgl. die finale Version: Das Europäische Parlament/Der Rat der Europäischen Union 2019) zu relativ großen Demonstrationen aufgerufen worden war, mutmaßte der CDU-Abgeordnete Daniel Caspary, die Proteste seien durch die Beteiligung gekaufter Demonstrant*innen so stark gemacht worden (vgl. Köver/Reuter 2019). Ergänzend vermutete der Europaabgeordnete Sven Schulze, Protestmails in derselben Sache seien von automatisierten Programmen (,Bots') abgeschickt worden (vgl. Kleinz 2019). 
2. FDP-Chef Christian Lindner verbreitete die folgende Aussage über die Schulstreiks im Zuge der Fridays for Future-Demonstrationen via Twitter: „Von Kindern und Jugendlichen kann man nicht erwarten, dass sie bereits alle globalen Zusammenhänge, das technisch Sinnvolle und das ökonomisch Machbare sehen. Das ist eine Sache für Profis." Daraufhin wurden von Kritiker*innen frühere Aussagen Lindners angeführt, die dazu in Widerspruch stehen (vgl. Disselhoff 2019).

3. Schließlich reagierte die Bundesvorsitzende der CDU, Annegret Kramp-Karrenbauer, im Anschluss an die Europawahlen auf das Rezo-Video ,Zerstörung der CDU“ mit Überlegungen zur Regulierung von ,Meinungsmache im digitalen Bereich“. Daraufhin wurde ihr vorgeworfen, die Meinungsfreiheit im Internet einschränken zu wollen. Dies sei ein Missverständnis, erwiderte sie.

Diese ,Missverständnisse und dieses Scheitern der Verständigung können als Hinweise darauf gedeutet werden, dass es einiger Anstrengung bedarf, um zwischen diesen verschiedenen Diskursrationalitäten zu vermitteln. Die Feststellung Engemanns (vgl. 2019), das Rezo-Video sei ein Indiz für das Aufkommen einer neuen Mündlichkeit, die eine Ablösung von einer dominanten Schriftkultur markiere, in Kombination mit der Bedeutsamkeit von Authentizität für jugendliche YouTube-Nutzer*innen, könnte zu einer neuen Form des ,miteinander Sprechens' anleiten. Als medial vermittelt sollte dieses Sprechen zumindest gedacht werden, wenn der Aussage stattgegeben wird, dass das Bewegtbild, also Video, als eine „Art Leitmedium“ zu begreifen sei (Netzwerk bewegtbildung.net 2019, S. 5), das diese neue Mündlichkeit erst ermöglicht.

Hierbei können wir sozusagen ,beruhigt' festhalten, dass Jugendliche (in Deutschland) an politischen Themen interessiert sind und YouTube oder andere Angebote im Internet dafür nutzen, sich zu informieren und sich ihre Meinung zu bilden. „Die haben eine große Neugier und eine große Energie, sie machen es nur halt anders." Seinen TwitterThread schließt Engemann mit einem Zitat der Band The Who von 1965: „The kids are alright.“ („Die jungen Leute sind in Ordnung.“; Engemann 2019) Die sozialen und medienkulturellen Formen, die dafür zum Zug kommen, weisen bei aller Innovation auch stets vertraute Aspekte aus älteren medialen Formen auf, die es erleichtern, eine gemeinsame Ebene (,Plattform‘?) der Verständigung zu finden.

Gleichzeitig wird in den bisherigen Untersuchungen deutlich, dass Jugendliche nicht immer auf allen Ebenen Problematiken von Plattformen wie YouTube durchdringen. Hugger et al. (vgl. 2019) stellen fest, dass Kritik etwa auf struktureller Ebene kaum zur Sprache kommt und auch die Differenzierung zwischen Information durch Intermediären wie YouTuber*innen oder klassischem Journalismus weitgehend oberflächlich 
bleibt. Entsprechend plädieren die Autor*innen für eine Verstärkung medienpädagogischer Medienkompetenzförderung im Bereich der Medienkritik, z.B. entlang der folgenden Fragen: Wie beurteile ich Quelle oder Inhalte als vertrauenswürdig? Welche Rolle spielen Algorithmen bei Vorschlägen für weitere Inhalte („User, die dieses Video gesehen haben, sahen auch...")?

Damit verbunden werden müsste die Frage nach dem Status von ,Expert*innen-Wissen“. Rezos CDU-Video, aber auch das Video-Statement der mehr als 90 You-Tuber*innen, setzt stark auf die Ansichten der scheinbar objektiven Expert*innen sowie der Wissenschaft allgemein. Was mit Bezug auf den Klimawandel eingängig erscheint, steht als pauschale Aussage der Perspektive entgegen, dass auch die Wissenschaft keinen monolithischen Wahrheitsanspruch erheben kann, sondern ebenfalls ein umkämpftes Feld verschiedener Paradigmen, Ansichten und normativer Lagerungen ist.

Was die Nutzung der politischen Arenen im Internet betrifft, sind nicht alle Parteien in Deutschland so weit von den dortigen Publika entfernt. Obwohl die Partei Alternative für Deutschland (AFD) in Umfragen davon profitiert, wenn sie die Aufmerksamkeit der klassischen Massenmedien erhält (vgl. am Beispiel von Zeitungen die Analyse von Hegelich/Papakyriakopoulos 2017), ist die AFD auf Facebook von allen deutschen Parteien mit Abstand am präsentesten und aktivesten: 1500 Seiten von Orts- und Kreisebene bis zur Bundesebene, 1,8 Mio. geteilte Beiträge alleine im März 2019 und damit mehr als sechs Mal mehr als alle anderen Parteien zusammen und ca. 4000 veröffentlichte Bilder pro Woche - schwerpunktmäßig zu den Themen Migration, Kriminalität, Klima und Gender. Einige Indizien lassen zwar auf einige halbautomatisierte Accounts in diesem Umfeld schließen, trotzdem erreicht die AFD mit ihren Online-Aktivitäten sichtlich sehr viele Menschen in Deutschland (vgl. pressesprecher Redaktion 2019).

Auch wenn der Schwerpunkt der AFD-Aktivitäten auf Facebook liegt, wird YouTube von AFD-nahen Personen genutzt. Der Verschwörungstheoretiker und ehemalige Wirtschaftsjournalist Oliver Janich, der selbst bereits durch die Verbreitung von Fake News aufgefallen ist (vgl. etwa Frerichmann 2018; Oswald 2018), rief 2017 in einem YouTube-Video zur Wahl der AFD auf (Oliver Janich 2017). Im Zuge der Debatten um das Rezo-Video veröffentlichte er eine Video-Einladung an die mehr als 90 YouTuber*innen, die den Nicht-Wahlaufruf unterstützt hatten, um mit ihm über Klimawandel zu diskutieren. Links zu diesem Aufruf, die von vielen verschiedenen Accounts gepostet wurden, füllen einen großen Teil der Kommentare unter ihrem VideoStatement. Auf diese Weise wird Janichs Einladung (und damit seine klimaskeptischen Positionen) nicht nur beworben, sondern es entsteht zugleich der Eindruck, diese Positionen würden von der Mehrheit der Menschen geteilt und unterstützt. 
Um faktisch ziemlich eindeutig falsche Aussagen über die Rolle und Verantwortung der Menschen im Klimawandel - aber auch verschwörungstheoretische, rassistische, sexistische und andere diskriminierende ,Meinungen " - auf YouTube, im Internet allgemein oder an der Bushaltestelle unten an der Straße nicht das Feld und damit die Deutungshoheit zu überlassen, bedarf es nicht nur der Anstrengung einer Altersgruppe, sondern einer gemeinsamen Positionierung von Jung und Alt - auf allen Kanälen.

\section{Literatur}

Anwar, Shanli (2019): Medienwissenschaftler über Rezo und Co. Die neue Neugier der Jugend. Christoph Engemann im Gespräch mit Shanli Anwar. Deutschlandfunk Kultur. Abgerufen unter: https://www.deutschlandfunkkultur.de/medienwissenschaftlerueber-rezo-und-co-die-neue-neugier.2156.de.html?dram:article_id=449853 [Stand vom 05-06-2019].

Berg, Achim (2017): Kinder und Jugend in der digitalen Welt. Berlin: bitkom. Abgerufen unter: https://www.bitkom.org/sites/default/files/file/import/170512-BitkomPK-Kinder-und-Jugend-2017.pdf [Stand vom 03-09-2019].

Bernau, Patrick; Záboji, Niklas; Carstens, Peter; et al. (2019): „Die Zerstörung der CDU": Das Rezo-Video im Faktencheck. FAZ Online. Frankfurt a.M. 24. Mai. Abgerufen unter: https://www.faz.net/1.6202603 [Stand vom 03-09-2019].

Bojanowski, Axel; Diekmann, Florian; Duhm, Lisa; et al. (2019): Rezo-Video „,Die Zerstörung der CDU“: Die Schwarzen getroffen? Spiegel Online, 24. Mai. Abgerufen unter: https://www.spiegel.de/politik/deutschland/rezo-video-die-youtube-angriffeauf-die-cdu-im-spiegel-faktencheck-a-1268973.html [Stand vom 03-09-2019].

Das Europäische Parlament \& der Rat der Europäischen Union (2019): Richtlinie (EU) 2019/790 über das Urheberrecht und die verwandten Schutzrechte im digitalen Binnenmarkt und zur Änderung der Richtlinien 96/9/EG und 2001/29/EG. Abgerufen unter: https://eur-lex.europa.eu/legal-content/DE/TXT/HTML/?uri=CELEX:32019 L0790\&from=EN [Stand vom 05-06-2019].

Disselhoff, Felix (2019): „, Sache für Profis “: FDP-Chef Lindner tadelt Schülerdemos - und stolpert über frühere Aussagen. meedia, Online Magazin. Abgerufen unter: https://meedia.de/2019/03/11/sache-fuer-profis-fdp-chef-lindner-tadelt-schuelerdemos-und-stolpert-ueber-fruehere-aussagen/ [Stand vom 08-06-2019].

Engemann, Christoph (2019): (1) Die Rückkehr der Vorlesung: ein paar medienwissenschaftliche Anmerkungenzu @rezomusik's Video.Thread. (1/18). @noisynarrowband, Tweet. Abgerufen unter: https:/twitter.com/noisynarrowband/ status/1131189722020241408?s=12 [Stand vom 03-09-2019]. 
Feierabend, Sabine; Rathgeb, Thomas; Reutter, Theresa \& Medienpädagogischer Forschungsverbund Südwest (Hrsg.) (2018): JIM-Studie 2018. Jugend, Information, Medien. Basisuntersuchung zum Medienumgang 12- bis 19-Jähriger. Stuttgart: Medienpädagogischer Forschungsverbund Südwest (mpfs). Abgerufen unter: https://www.mpfs.de/fileadmin/files/Studien/JIM/2018/Studie/JIM2018_Gesamt.pdf [Stand vom 03-09-2019].

Fisher, Max \& Taub, Amanda (2019): On YouTube's Digital Playground, an Open Gate for Pedophiles. The New York Times, 4. Juni. Abgerufen unter: https://www.nytimes.com/2019/06/03/world/americas/youtube-pedophiles.html [Stand vom 03-092019].

Frerichmann, Nora (2018): Das Altpapier am 17. August 2018: Verteidigt oder verrannt? mdr.de: Das Altpapier. Abgerufen unter: https://www.mdr.de/altpapier/dasaltpapier-604.html [Stand vom 03-09-2019].

Gebel, Christa; Jünger, Nadine \& Wagner, Ulrike (2013): Online-Mediengebrauch Jugendlicher. Umgang mit gesellschaftlich relevanter Information. In: merz medien+erziehung -zeitschrift für medienpädagogik, (2013/03), S. 33-41.

Gräßer, Lars; Hugger, Kai-Uwe \& Kaspar, Kai (2019): Peers und politische Orientierung von Jugendlichen. YouTuber-Videos (2017) | Grimme-Forschungskolleg, Projekt-Website. Abgerufen unter: https://www.grimme-forschungskolleg.de/portfolio/ youtuber-videos-2017/ [Stand vom 03-09-2019].

Hegelich, Simon \& Papakyriakopoulos, Orestis (2017): Zwischen \#AfDErfolg und Medienberichten gibt es einen kausalen Effekt - und der lässt sich berechnen. Political Data Science, Science Blog. Abgerufen unter: http://politicaldatascience.blogspot. com/2017/09/zwischen-afderfolg-und-medienberichten.html [Stand vom 03-092019].

Hugger, Kai-Uwe; Braun, Lea; Noll, Christian; et al. (2019): Zwischen Authentizität und Inszenierung: Zur medienkritischen Einschätzung informationsorientierter YouTuber*innen-Videos durch Jugendliche. In: von Gross, Friederike; Röllecke, Renate (Hrsg.) Instagram und YouTube der (Pre-)Teens. Inspiration, Beeinflussung, Teilhabe, München: kopaed, S. o.S.

Hugger, Kai-Uwe; Kaspar, Kai \& Gräßer, Lars (2018): YouTuber*innen-Videos und politische Orientierungen von Jugendlichen. Bielefeld: Universität zu Köln, GrimmeForschungskolleg, Grimme Institut. Abgerufen unter: https://www.gmk-net.de/wpcontent/uploads/2018/06/nrw-fachtagung_influencer_impuls_folien_hugger_noll. pdf [Stand vom 03-09-2019].

Institut für Protest- und Bewegungsforschung (ipb) (2019): Fridays for Future. Eine neue Protestgeneration? Ergebnisse einer Befragung von Demonstrierenden am 15. März 2019 in Berlin und Bremen. Berlin. Abgerufen unter: https://protestinstitut.eu/ 
erste-ergebnisse-der-befragung-der-fridays-for-future-proteste/ [Stand vom 03-092019].

Kleinz, Torsten (2019): ,Wir sind die Bots “ - über 1000 demonstrieren gegen Artikel 13. heise online. Abgerufen unter: https://www.heise.de/newsticker/meldung/Wirsind-die-Bots-ueber-1000-demonstrieren-gegen-Artikel-13-4311105.html [Stand vom 03-09-2019].

Köver, Chris \& Reuter, Markus (2019): „, Gekaufte Demonstranten “: Die Strategie der Autoritären zur Diskreditierung von Protest. netzpolitik.org. Abgerufen unter: https://netzpolitik.org/2019/gekaufte-demonstranten-die-strategie-der-autoritaerenzur-diskreditierung-von-protest/ [Stand vom 03-09-2019].

Kupferschmitt, Thomas (2018): Onlinevideo-Reichweite und Nutzungsfrequenz wachsen, Altersgefälle bleibt. Ergebnisse der ARD/ZDF-Onlinestudie 2018. In: Media Perspektiven, (9), S. 427-437. Abgerufen unter: www.ard-zdf-onlinestudie.de/ files/2018/0918_Kupferschmitt.pdf [Stand vom 03-09-2019].

Leven, Ingo \& Schneekloth, Ulrich (2015): Freizeit und Internet: Zwischen klassischem »Offline« und neuem Sozialraum. In: Albert, Mathias; Hurrelmann, Klaus; Quenzel, Gudrun; et al. (Hrsg.) Jugend 2015. Eine pragmatische Generation im Aufbruch, Frankfurt am Main: Fischer Taschenbuch, (Shell-Jugendstudie), S. 111-151. maiLab (2019): Rezo wissenschaftlich geprüft. YouTube-Video. Abgerufen unter: https://www.youtube.com/watch?v=tNZXy6hfvhM [Stand vom 03-09-2019].

Netzwerk bewegtbildung.net (2019): Bewegtbildung denken - Beiträge zu Webvideo und politischer Bildung im Social Web. Berlin: bpb; mediale pfade.org. Abgerufen unter: http://bewegtbildung.net/wp-content/uploads/2019/05/Bewegtbildung_denken_\%E2 \%80\%93_Beitraege_zu_Webvideo_und_politischer_Bildung_im_Social_Web.pdf [Stand vom 03-09-2019].

Oliver Janich (2017): Warum Sie die Alternative für Deutschland (AfD) wählen sollten. Oswald, Bernd (2018): Was ist dran an Gerüchten zu angeblichem Wahlbetrug? BR24. Abgerufen unter: https://www.br.de/nachrichten/bayern/was-ist-dran-an-geruechten-zu-angeblichem-wahlbetrug,R6pR9h3 [Stand vom 03-09-2019].

pressesprecher Redaktion (2019): AfD mit $85 \%$ aller Parteien-Shares bei Facebook. Magazin pressesprecher. Abgerufen unter: https://www.pressesprecher.com/nachrichten/afd-mit-85-aller-parteien-shares-bei-facebook-1778839550 [Stand vom 0506-2019].

Rakebrand, Thomas \& Jünger, Nadine (2013): Das Internet als Informationsmedium Jugendlicher. Informationsbezogene Aneignung von Online-Angeboten am Beispiel Musik. In: merz - medien+erziehung - zeitschrift für medienpädagogik, (2013/03), S. 25-32. 
Rat für Kulturelle Bildung (2019): Jugend / YouTube / Kulturelle Bildung. Horizont 2019. Eine repräsentative Umfrage unter 12- bis 19-Jährigen zur Nutzung kultureller Bildungsangebote an digitalen Kulturorten. Essen: Rat für Kulturelle Bildung (RKB). Abgerufen unter: https://www.rat-kulturelle-bildung.de/fileadmin/user_upload/pdf/Studie_YouTube_Webversion_final.pdf [Stand vom 03-09-2019].

rezo (o.J.): rezo - Kanalinfo. YouTube - Kanalinfo, Videoplattform. Abgerufen unter: https://www.youtube.com/channel/UCLCb_YDL9XfSYsWpS5xrO5Q/about [Stand vom 05-06-2019].

Rezo ja lol ey (2019a): Die Zerstörung der CDU. YouTube-Video. Abgerufen unter: https://www.youtube.com/watch?v=4Y11ZQsyuSQ [Stand vom 03-09-2019].

Rezo ja lol ey (2019b): Ein Statement von 90+ Youtubern. YouTube-Video. Abgerufen unter: https://www.youtube.com/watch?v=Xpg84NjCr9c [Stand vom 03-09-2019]. Rummler, Klaus \& Wolf, Karsten D. (2012): Lernen mit geteilten Videos: aktuelle Ergebnisse zur Nutzung, Produktion und Publikation von Onlinevideos durch Jugendliche. In: Sützl, Wolfgang; Stalder, Felix; Maier, Ronald; et al. (Hrsg.) Media, knowledge and education: cultures and ethics of sharing. Innsbruck: Innsbruck University Press, S. 253-266. Abgerufen unter: https://www.uibk.ac.at/iup/buch_ pdfs/9783902811745.pdf [Stand vom 03-09-2019].

Statista (2018): Online-Videos - Wöchentliche Nutzung nach Altersgruppen in Deutschland 2018. Statista. Abgerufen unter: https://de.statista.com/statistik/daten/studie/958878/umfrage/woechentliche-nutzung-von-online-videos-nach-altersgruppen-in-deutschland/ [Stand vom 05-06-2019].

Statistisches Bundesamt (2018): Bevölkerung im Alter von 65 Jahren und mehr. Basistabelle nach Daten von World Development Indicators und Weltbank. destatis.de. Abgerufen unter: https://www.destatis.de/Migration/DE/ZahlenFakten/LaenderRegionen/Internationales/Thema/Tabellen/Basistabelle_Bevoelkerung65.html [Stand vom 05-06-2019].

Strobl, Richard (2019): Europawahl 2019: Grüne holen Erdrutschsieg bei jungen Wählern - So unterschiedlich wählten Frauen und Männer. merkur.de, 27. Mai. Abgerufen unter: https://www.merkur.de/politik/europawahl-2019-wer-hat-wengewaehlt-nach-alter-geschlecht-bildung-und-beruf-zr-12322655.html [Stand vom 03-09-2019].

tagesschau (2019): „Zerstörung der CDU“: Amthor zur fehlenden Videoantwort der CDU auf Rezo. YouTube-Video. Abgerufen unter: https:/www.youtube.com/ watch? $\mathrm{v}=$ DEKzmztBZNI [Stand vom 03-09-2019].

tagesschau.de (о.J.): Umfragen Wähler nach Altersgruppen. tagesschau.de. Abgerufen unter: https://wahl.tagesschau.de/wahlen/2019-05-26-EP-DE/umfrage-alter.shtml [Stand vom 05-06-2019]. 
Wolf, Karsten D. \& Kratzer, Verena (2015): Erklärstrukturen in selbsterstellten Erklärvideos von Kindern. In: Hugger, Kai-Uwe; Tillmann, Angela; Iske, Stefan; et al. (Hrsg.) Jahrbuch Medienpädagogik 12. Kinder und Kindheit in der digitalen Kultur, Wiesbaden: Springer Fachmedien Wiesbaden, (Jahrbuch Medienpädagogik), S. 2944, doi: 10.1007/978-3-658-09809-4_3. 